\section{Hard-Copy Imaging Options}

\section{Leonard Polizzotto, Polaroid Corporation}

Photographic film has played a central role in the recording of microscope images since the invention of photography in the 1830 s. Photography rapidly creates an accurate record of the microscopic specimen and avoids the subjectivity that was inherent in written and artistic descriptions of visual observations. Both instant and conventional films offer high resolution, the ability to record a large tonal gradation, and a stable media for long-term data storage.

Newer techniques, such as thermal, ink jet, and laser prints, have grown in popularity because they offer ease-of-use or lower per-image cost. Despite recent improvements, none of these techniques offer the resolution or stability of a photograph. In general, people have been forced to sacrifice quality and permanence in order to achieve the lower per-image cost and ease-of-use promised by alternative recording systems.

Photographic hard copy has traditionally served as both the communication and storage medium. The introduction of digital storage methods has made these functions more distinct. Therefore, it is important to understand the trade offs between hard copy images and electronically stored images Magnetic, optical, and solid state electronic storage technologies differ significantly in their cost per image, time and ease to record and retrieve, and display flexibility. Photographic hard copy continues to offer some archival storage advantages, and hard copy remains a requirement for reports, presentations, and discussions.

Each of the current hard copy recording options offers a mix of advantages and disadvantages. The ideal hard copy medium for microscopy would combine the quality and permanence of photography with the price and easeof-use of the alternative systems. This ideal hard copy system would also offer the correct contrast and dynamic range, so that all the important details in the micrograph will be visible within the print. A black-and-white system

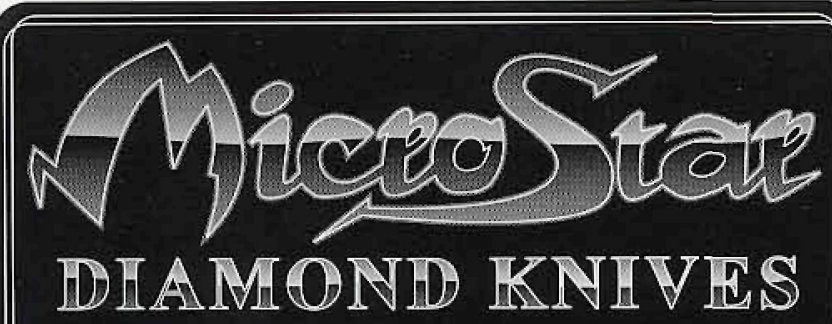

QU ANLITS

Backed by an iron clad guarantee: you don't pay until you have tested your knife and are totally satisfied with its flawless quality. BBIST SHIFCTHON

Choose from 8 boat styles, 15 types and 59 sizes, from 1 to $10 \mathrm{~mm}$. BIEST SIERVICHS

Exchange (or resharpen) your old knife, any brand, type, size or age for a new MICRO STAR at the resharpening price.

RT 2 BOX 474, HUNTSVILLE TX 77340 USA TEL 4092916891 FAX 4092949861 BDO 5332509 would be able to record a full range of gray values with rich whites and blacks, and a color system would be able to record a full range of colors.

In recent years, Polaroid Corporation has developed a variety of black-andwhite and color imaging media that are activated by diode lasers. Unlike existing laser printers that produce an image by fusing toner to a paper or transparent base, these new imaging systems use laser power to print directly on the new media by creating phase transitions within it. The new laser imaging systems show promise for eliminating the current trade offs between cost, ease-of-use, quality, and permanence. The instant processes require no running water or wet chemistry, the imaging time is short, and the prints offer long term archival permanence.

A black-and-white laser imaging system was demonstrated at the "Future Directions in Microscopy and Imaging" workshop held before the 1992 EMSA Meeting. This system has now been beta tested at a variety of large scientific imaging locations with outstanding results.

The new plack-and-white process uses carbon particles instead of photographic silver and relies upon high power laser diodes to write the image. The film consists of a 7 mil polyester base, a laser sensitive layer, an imaging layer composed of carbon particles in a polymeric matrix, and a cover sheet.

The film is exposed with high-power gallium-arsenide solid state laser diodes that write a high-resolution image. When the laser strikes the film, its energy is absorbed by the laser sensitive layer. The absorbed energy causes a phase transformation that creates a permanent adhesion of the imaging layer to the laser sensitive layer. Following exposure, the cover sheet is peeled off, which removes the unactivated portions of the imaging layer. This leaves the positive image permanently affixed to the substrate.

There are some important variables that must be carefully controlled to produce a high-quality image with this new technique. The carbon particles must be small and have a narrow size distibution in order to create a uniform spot size. And the laser energy must be applied for very brief intervals - typically a few hundred nanoseconds - to prevent the energy from affecting areas that should remain unexposed. In addition, the separation of the film sandwich has to be carefully controlled, to ensure that all unexposed areas are removed and all exposed areas are kept in place.

The new laser imaging system produces a full range of grays by using a digital gray-scale rendering technique that subdivides each of the individual pixels in 4320 separate pels. Each pel is individually exposed by focusing the laser beam onto a small area of the film that is only a few microns square. The large number of individual pels per pixel creates a film with 4320 or 12 bits of gray value. The 4320 potential gray values in this laser printing system make it possible to precisely define any film response curve, by simply adjusting the subset of actual values that are used for a particular picture.

The new laser medium matches photographic permanence, because there are no fadeable dyes, such as those used in the thermal transfer and dye diffusion process. Instead, the image is composed of stable carbon particles that are permanently affixed to the polyester base by an irreversible phase transformation.

Color images are becoming increasingly important in scientific imaging and a new color laser imaging method is also under development at Polaroid. This system uses laser energy to activate color dyes within the medium. The laser exposure transforms the medium into a full-color slide, reflective print, or overhead transparency.

While the black-and-white laser imaging system requires only a single laser wavelength, the new color system uses lasers with three different infrared wavelengths to create phase tansitions for yellow, cyan, and magenta dyes in the single sheet, completely dry media. This new imaging system combines the resolution and permanence of photography with the ease-of-use and lower perimage cost of other current imaging methods. It is expected that the new color system will find many applications in microscopy and scientific imaging.

Reprinted from Proceedings, 51st Meeting of the Microscopy Society of America with the permission of MSA. 